\title{
ORGANIZACIONES INDÍGENAS, POLÍTICAS PÚBLICAS Y PROYECTOS DE DESARROLLO EN LA LOCALIDAD DE PAMPA DEL INDIO, PROVINCIA DE CHACO, ARGENTINA
} Indigenous Organizations, Public Policies and Development Projects in the Locality of Pampa del Indio, Province of Chaco, Argentina

\section{MALENA CASTILLA*}

Fecha de recepción: 30 de diciembre de 2017 - Fecha de aprobación: 09 de abril de 2018

\section{Resumen}

El trabajo que aquí presentamos busca dar cuenta del proceso de conformación y consolidación de las organizaciones indígenas del pueblo qom ubicadas en la localidad de Pampa del Indio, provincia de Chaco. Intentaremos, además, dar cuenta de este proceso a partir del análisis sobre las articulaciones y tensiones que se presentan entre dichas organizaciones étnicas y otras en los procesos de implementación de políticas públicas y proyectos de desarrollo. Por ello, para describir estos procesos en el presente artículo haremos un recorte temporal, que abarca desde el año 2001 hasta la actualidad, con el objetivo de abordar las relaciones que existieron entre los diversos actores -organizaciones étnicas, las Organizaciones No Gubernamentales, los organismos de financiamiento gubernamentales e internacionales- a partir de la implementación de diferentes políticas públicas en el territorio.

Palabras clave: organizaciones indígenas; políticas públicas; organizaciones no gubernamentales; organismos internacionales.

\section{Abstract}

The work we present here seeks to account for the process of conformation and consolidation of the indigenous organizations of the qom people located in the town of Pampa del Indio, Chaco province. We will also try to give an account of this process from the analysis of the articulations and tensions that are presented between these ethnic organizations and others in the processes of implementation of public policies and development projects. For this reason, to describe these processes in this article we will make a temporary cut, which covers from the year 2001 to the present day, with the aim to address the relations that existed between the diverse actors-ethnic organizations, the Non-governmental organizations, governmental and international agencies, based on the implementation of different public policies in the territory.

Keywords: indigenous organizations; public policies; non-governmental organizations; international organizations.

\footnotetext{
* Becaria doctoral por la Universidad de Buenos Aires. Doctoranda Facultad de Filosofía y Letras, Universidad de Buenos Aires, Buenos Aires, Argentina. El artículo se enmarca en su tesis doctoral. Correo-e: malenacastilla@gmail.com
} 


\section{Introducción}

Notoriamente, en las últimas décadas se evidencia un incremento en la cantidad de integrantes de los pueblos indígenas, a pesar de diferentes hechos que marcaron una disminución en la población, sobre todo de pueblos como el qom. Dicho pueblo, históricamente, tuvo una presencia altamente significativa en la República Argentina. Entre estos hechos que marcaron la disminución de la población estuvieron: la consumación de la conquista de la región chaqueña al mando del General Victorica a fines del siglo XIX, la cual dejó como resultado pueblos y aldeas indígenas derrotadas, como afirman Bartolomé (2003) y Trinchero (2009). Posteriormente durante el periodo del Siglo XX "Como consecuencia de las profundas transformaciones socioeconómicas" se "han producido grandes oleadas migratorias" hacia los diferentes centros urbanos del país y de países limítrofes (Weiss, Engelman \& Valverde, 2013, p. 02).

No obstante, dichos sucesos, el pueblo qom ha aumentado cuantitativamente en los últimos años, como mencionamos anteriormente, posiblemente, debido a un "reconocimiento de la pluralidad étnica y lingüística en el país" (Hecht, 2014, p. 02). Cabe destacar, que dicho pueblo cuenta con más de $100 \mathrm{mil}$ habitantes que se auto reconocen como miembros del pueblo qom según el Instituto Nacional de Estadísticas y Censos (INDEC, 2010). Particularmente, la provincia de Chaco, que nos ocupa en este trabajo, posee un 3,9\% de población indígena. Se trata de algo más de 40.000 integrantes de los pueblos originarios qom (toba), mocoví, wichí, guaraní, diaguita calchaqui, atacama y quechua.
Pampa del Indio es una localidad ubicada al norte de la provincia de Chaco, en el departamento Libertador General San Martín. Según el Censo realizado por el INDEC, en el año 2001 vivían en dicha localidad 11.558 habitantes y en los datos del último censo del año 2010, la población registrada fue de 13.675 personas. Más de la mitad de este porcentaje vive en parajes de entre 700 y 800 habitantes, el resto de la población reside en parajes con menos de 350 personas, es decir, en los montes de la región (INDEC, 2001, 2010). Cabe mencionar que, en esta localidad, solo se encuentran dentro de los habitantes indígenas integrantes del pueblo qom. En este sentido el pueblo qom, atravesado por procesos que han influenciado significativamente en el accionar de dicha población -como es el caso de: la colonización (como mencionamos anteriormente), "el despliegue de intervenciones para el desarrollo que comprenden políticas de manejo de los recursos naturales presentes en el ámbito del frente expansivo regional" (Iñigo Carrera, 2012b, p. 2), la producción algodonera y posterior sustitución con la soja (Leoni de Rosciani, 1992), entre otros acontecimientos- han logrado organizarse en "movimientos etnopolíticos" (Bartolomé, 2004), reconocidos nacional e internacionalmente, ya sea por las luchas que llevan a cabo por la restitución de sus tierras, por las medidas que llevan a cabo las comunidades acerca del cumplimiento de sus derechos como pueblo indígena (Carrasco \& Briones, 1996) o por los reclamos para acceder a un sistema educativo intercultural (Hecht, 2006), entre otras cosas. 


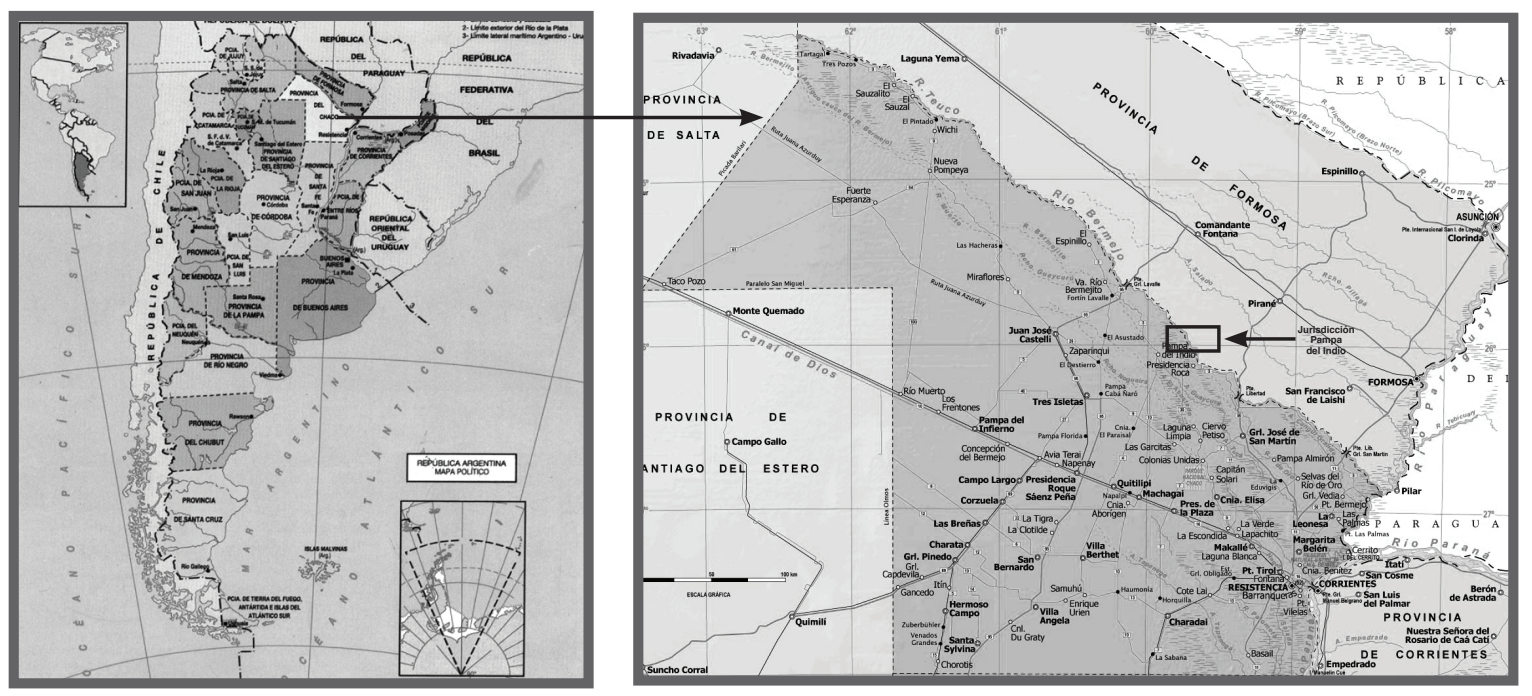

En la primera imagen se observa el mapa de Argentina y se amplifica la imagen correspondiente a la provincia de Chaco (segunda imagen). En esta última una flecha señala la ubicación de la localidad de Pampa del Indio. Imagen disponible en: www.mapade.org (fecha de consulta 10 de abril de 2018). Imagen de la provincia de Chaco tomada del Instituto de Educación Superior de Pampa del Indio.

Disponible en: https://inspampadelindio-cha.infd.edu.ar (fecha de consulta: 10 de abril de 2018)

El incremento de la población indígena según los datos del censo de los años 2001 y 2010 se debe a un proceso de visibilización y reactualización entre esta población en Argentina. En primer lugar, consideramos que este proceso se enmarca en un contexto donde las políticas públicas y los organismos de financiamiento gubernamentales e internacionales han puesto la atención en dichos pueblos a la hora de pensar líneas de acción en las últimas décadas. Por otro lado, creemos que tal proceso también podría explicarse en función de la presencia que se ha establecido a nivel nacional acerca de las demandas, conflictos y reivindicaciones vinculadas a los pueblos indígenas. Estos hechos dan cuenta que las realidades, sumamente complejas, en las cuales las comunidades étnicas se vinculan, lejos de hacerlas desaparecer o "anular la referencia al origen, lo refuerzan" a partir de un proceso de actualización (Oliveira Pacheco, 2010, p. 26). Cabe aclarar que dicho proceso no ocurrió en Argentina solamente (Bartolomé, 2008; Carrera, 2012; García \& Valverde, 2007; Radovich \& Balazote, 2009; Trinchero, Muñoz \& Valverde, 2014), sino también en toda América Latina (Bengoa, 2000, 2009; Bello, 2004; Campos, 2006; Espinoza 2017; Stavenhagen, 2005; Valverde, 2013).

En este contexto de reactualización indígena a partir de la influencia etnopolíticas en el campo social, este artículo tiene como objetivo analizar los procesos, las articulaciones y tensiones a partir de los cuales se han consolidado y afirmado las organizaciones indígenas en la localidad de Pampa del Indio. En este sentido, centraremos la atención en el período temporal que abarca los años desde el 2001 (dada que 
esta es una fecha clave en los últimos años en Argentina) hasta la actualidad. Por tanto, analizaremos la manera en que las organizaciones indígenas locales se han consolidado en el tiempo y los modos en que sus integrantes se han convertido en sujetos claves a partir de la vinculación y el trabajo generado por la implementación de políticas públicas nacionales e internacionales. En este sentido retomaremos el análisis de diferentes autores como Trinchero y Leguizamón (1995), De la Cruz (1997), Braticevic (2011) -entre otra vasta literatura- que han analizado el rol de organismos de financiamiento internacional en la implementación de políticas en localidad de la región chaqueña, que buscan alcanzar a las poblaciones indígenas.

Asimismo, nos resulta importante destacar que este trabajo se enmarca en una investigación doctoral en Antropología Social, elaborada en del trabajo de campo en la localidad de Pampa del Indio. Es decir, este artículo forma parte de un proyecto de investigación más amplio que tiene como objetivo general describir y analizar los procesos de conformación y consolidación de las organizaciones indígenas con las que aquí nos proponemos trabajar en relación a la interacción que mantienen con diferentes agentes públicos y privados.

La metodología utilizada fue la etnografía y las técnicas aplicadas, las entrevistas en profundidad, así como entrevistas semi abiertas, individuales y grupales, a diferentes miembros de organizaciones indígenas y organizaciones no gubernamentales. La observación participante, las situaciones conversacionales y el trabajo con fuentes periodísticas también fueron parte de las herramientas utilizadas para elaborar este documento. Cabe mencionar que a lo largo del trabajo de campo se han realizado nume- rosas entrevistas y conversaciones informales con los integrantes de la comunidad étnica, asi como miembros de fundaciones, organismos no gubernamentales y funcionarios estatales. Dadas las limitaciones de espacio en este artículo, solo retomamos algunos fragmentos que encontramos más significativos de las entrevistas para representar la problemática aquí analizada. Es necesario aclarar que los nombres de los entrevistados, actores, fundaciones y organizaciones que figuran en este escrito, fueron reemplazados por nombres ficticios para preservar su anonimato.

\section{La organización social e indígena frente a la crisis de 2001}

En el año 2001 se experimentó una fuerte crisis económica, social y política, que tuvo su pico de ebullición en las últimas semanas del mes de diciembre, con protestas sociales y represión policial, producto del paquete de medidas neoliberales que se venían implementando fuertemente desde la década anterior, tales como: la desregulación el sector financiero, la implementación de leyes de flexibilización laboral y la privatización de empresas como la compañía estatal petrolera YPF o Aerolíneas Argentinas, además de la venta indiscriminada de tierras al capital privado transnacional en todo el territorio argentino nacional, entre otras (Castilla, 2017). Finalmente, el 2 de enero del 2002, quien ocupó el cargo de presidente fue Eduardo Duhalde elegido por el Poder Legislativo siguiendo el reglamento de la Ley de Acefalía-, hasta mayo del 2003 cuando asume la presidencia Néstor Carlos Kirchner mediante el voto popular.

La gestión de Kirchner significó un cambio en materia económica y justicia social. El camino 
a la recomposición social era arduo y la población necesitaba medidas extremas para salir de la condición de pobreza. En el marco de la declaración de la emergencia alimentaria, ocupacional y sanitaria, se creó -ya en la gestión de E. Duhalde (2002-2003)- a partir del decreto 565/021 del año 2002, el "Programa Jefes de Hogar", también denominado "Derecho Familiar de Inclusión Social: Plan Jefes y Jefas Desocupados”. Dicho programa alcanzó a dos millones de beneficiarios de todo el país sobre más de 36.260 .130 habitantes (INDEC, 2001). Para poder cobrar el subsidio las condiciones eran: no tener un trabajo, ser jefe o jefa de hogar y tener hijos en edad escolar. El plan otorgaba una tarjeta de débito, siendo esta iniciativa una propuesta de los bancos, las empresas y el Banco Mundial. Dicha propuesta dejaba de lado a aquellos comercios que no estuviesen bancarizados. Por tanto, este plan no contribuía a la reactivación económica interna, pero representaba, sin embargo, una gran ayuda para las familias que no tenían ningún recurso. Es decir, las organizaciones no gubernamentales y organismos como el Banco Mundial, continúan invirtiendo en el país a partir de la aplicación de políticas de desarrollo, usufructuando la situación económica y social (Acuña, 2014; Braticevic, 2011).

Estos subsidios se comenzaron a entregar en toda la región chaqueña, y muchos hombres y mujeres los cobraron en Pampa del Indio en particular. La contraprestación que tenían que realizar por dicho ingreso era el mantenimiento de la vía pública, ya sea limpiando las calles o las dependencias gubernamentales. Las mujeres además trabajaban en comedores infantiles o merenderos. En una entrevista a Andrea ${ }^{1}$, integrante de 'Las Tejedoras', nos cuenta cómo fue el paso de esta organización por el programa:
Por allá cuidabamos chiquitos acá y en tiempo crítico, en 2002, 2001, se implementó el 'Jefes de Hogar' y las mamás tenían que cumplir tareas obligatorias en el hogar para cobrar, y nos pagaban por cuidar a los chiquitos y nos turnábamos, hasta los cuatro años, porque los de cinco iban al jardín. Les dábamos la leche con la ayuda de las hermanitas. (Andrea, Pampa del Indio, abril del 2016)

Durante esta década la 'patria sojera'2 había avanzado sobre el $59 \%$ del territorio nacional, lo cual significó la apropiación de tierras, los conflictos por el uso de agroquímicos y la incidencia en la salud de los habitantes y del medioambiente, así como el enriquecimiento de una porción minoritaria de la población a costa del perjuicio del sector mayoritario (Aranda, 2012). Este enriquecimiento de los grandes empresarios devino en el año 2008 en el conocido 'conflicto del campo', en el que se produjo un lock out de las patronales agropecuarias contra el gobierno nacional de la entonces presidenta Cristina Fernández de Kirchner (2007-2011 y 2011-2015), quien tomaría medidas que establecían un sistema de retenciones impositivas a la soja, el trigo y el maíz. Dicha medida encontró una fuerte oposición en diferentes organizaciones patronales como la Sociedad Rural Argentina, la Confederación Rural Argentina, la Confederación Intercooperativa Agropecuaria Cooperativa Limitada y la Federación Agraria Argentina, que presentaron distintas medidas de lucha contra la resolución gubernamental. Sin embargo, esta medida de los empresarios del agro no reflejaba la postura que mantenía otra parte de los trabajadores del campo, los pequeños productores. Entre las organizaciones que trabajaban en Chaco, y específicamente en Pampa del Indio, los integrantes de la Unión de Pequeños Productores de Chaco (UNPEPROCH) se manifestaron en contra de 
la medida de sus colegas más poderosos. Esta acción generó en el año 2008 la creación de los 'Consorcios Productivos de Servicios Rurales'- entidades de bien público de servicio a la comunidad, sin fines de lucro, conformados por pequeños productores que poseían hasta 50 hectáreas- en toda la provincia chaqueña con el fin de empoderar a los pequeños productores locales y "aunar esfuerzos y aportes económicos de distinta naturaleza para lograr la contención, el desarrollo y el fortalecimiento de los pequeños productores chaqueños" (Ley $6547 / 08$ de la Provincia de Chaco). En esta ley, además, se los exceptuaba del pago de tasas e impuestos provinciales. La creación de los consorcios era la respuesta a un reclamo que las organizaciones de pequeños productores comenzaron a realizar desde el corrimiento de la frontera sojera y el reemplazo de la producción de algodón por soja. Asimismo, no solo los pequeños productores criollos eran los que se veían beneficiados con esta medida sino también aquellos indígenas que trabajaban en el agro y eran parte de los consorcios rurales en la localidad de Pampa del Indio.

Ahora bien, las organizaciones étnicas que no se reconocían con una identidad campesina o productora, y que siguieron trabajando, se consolidaron en esta etapa y continuaron reclamando por el cumplimiento de sus derechos indígenas. Cabe mencionar, que este proceso de organización se verifica en América Latina durante esta etapa, donde los pueblos indígenas se consolidan en tanto sujeto de derecho y se conforman como movimientos sociales (Bello \& Ranger, 2002, 2004; Blanco, 2005; Stavenhagen, 2002). Este fue uno de los ejes que motivó a aquellas organizaciones a intervenir junto a las ONGs como son el caso del Equipo Nacional de Pastoral Aborigen (ENDEPA), el Insti- tuto de Cultura Popular (INCUPO), entre otras. ENDEPA e INCUPO, son instituciones del orden de la religión católica que se emplazaron en el territorio de Pampa del Indio durante la década de 1980 y siguen teniendo vigencia y relevancia en la actualidad. Estas organizaciones se dedicaron a trabajar con las comunidades indígenas de la región a partir de diferentes tareas de acompañamiento y asesorías (Castelnuovo, 2016). Los reclamos de dichas agrupaciones se centraban en el reconocimiento cultural, la educación intercultural, su preexistencia y la posesión de las tierras (notas de trabajo de campo de la autora, noviembre 2016).

El contexto de crisis, si bien insertó en una pobreza más abrupta a este sector de la población, también generó otros efectos, como un mayor acercamiento y unión entre los habitantes. En este sentido, las integrantes de la organización étnica 'Las Tejedoras' comenzaron a cumplir un rol social dentro de la comunidad más allá de la tarea específica asignada por el plan Jefes de Hogar (darles la merienda y cuidar a los niños):

Acá habíamos armado una cuna para los nenitos, el idioma nuestro ellos lo entendían y les hacíamos cantar. Con el correr del tiempo crecieron y fueron a la escuela y como que ya no era tan obligatorio el Jefe de Hogar comenzamos a escribir los cuentos. Nos dimos cuenta de que los chicos estaban perdiendo la cultura nuestra y teníamos que recuperar las tradiciones, por eso surgió el nombre de Madres Cuidadoras y en ese momento la hermana Magdalena nos apoyó en lo que estábamos haciendo y salió todo positivo. (Andrea, Pampa del Indio, abril 2016)

"Las Tejedoras" habían pasado de ser costureras de su vestimenta y la de sus familias, a ser promotoras de la preservación cultural, que se veía afectada por los contextos antes 
descritos, además de la historia nacional de negación hacia las poblaciones indígenas. En este escenario, y a partir de su trabajo en la comunidad con la participación en el Plan Jefes de Hogar, comenzaron a denominarse "Huo'o ra lyac na alpi cha'aye retelataxan 'alpi” -en castellano significa: Hermosas Mujeres que Cuidan (de ahora en más Huo'o)-. Cabe aclarar que esta organización étnica comenzó sus trabajos a partir de la vinculación con el Equipo Nacional de Pastoral Aborigen (ENDEPA) y el Instituto Nacional de Cultura Popular (INCUPO). Durante el año 2003 presentaron un proyecto a la Orden de la Merced $^{3}$ y comenzaron a recibir un subsidio para trabajar en pos de la recuperación cultural. Es a partir de este año que empezaron a redactar sus objetivos y a formalizarse en tanto organización, con el fin de obtener subsidios para su funcionamiento. El financiamiento que les fue otorgado por la Orden de la Merced incluía un ingreso individual para cada integrante en calidad de beca y un monto para la impresión de láminas con dibujos y leyendas propias de su cultura, las cuales utilizan hasta la actualidad en cada actividad que realizan. Alejandra, integrante del Área de Acción Mercedaria de la Orden, nos contaba en una entrevista las primeras acciones realizadas con la organización indígena:

Se arranca a financiar la merienda de los chicos y después en 2004 se crea esta área de Acción, y a partir de ahí ellas siguen presentando por etapas los proyectos que tenían. Hacen tres etapas por año y se iban transfiriendo los fondos, y así se hizo acompañamiento, y por medio de ese proceso ellas hicieron los dibujos y láminas donde muestran los animales, las leyendas, las tradiciones para enseñar a los niños y la gente de la comunidad, y cuando surgían cosas que no podían cubrir, las hermanas se comunicaban diciendo si había posibilidad de transferir y se hizo el libro. (Alejandra, Orden de la Merced, abril 2016)
Además del trabajo con la Orden, los integrantes de la organización Huo'o comenzaron a trabajar con un equipo de extensión de la Universidad del Nordeste dirigido por la profesora Leiras. Dicho equipo buscaba investigar en Pampa del Indio el trabajo que llevaban a cabo las organizaciones respecto a la educación intercultural. A partir de este contacto, la organización étnica comenzó a vincularse con miembros de la mencionada Universidad para la realización de diferentes actividades de formación, para sus propios integrantes como para los mismos alumnos universitarios que trabajan en el equipo de Leiras; estos últimos viajaban desde el año 2007 a la localidad para trabajar en el territorio, además de llevar a cabo jornadas en la universidad vinculadas a la educación bilingüe intercultural y a la cuestión indígena.

Asimismo, la organización Huo'o forma parte del consejo de organizaciones indígenas denominada 'Enauac, que se ha consolidado en la década de 1990. Desde ese momento la organización trabaja con comunidades étnicas de Pampa del Indio, y en el año 2000 adquirieron un total de 104 hectáreas para ser utilizadas por los indígenas de la región en la construcción de un complejo educativo intercultural. Dicho complejo fue inaugurado más tarde, en el año 2012. Cabe resaltar que el 'Enauac trabaja, ante todo, en la reglamentación de la ley nacional respecto a la educación intercultural.

La visibilidad alcanzada por las organizaciones étnicas en la localidad de Pampa del Indio y la posibilidad de dar a conocer sus reclamos (acerca de la aplicación de las leyes y el respeto cultural) se generó, entre otros hechos, a partir de su participación en diferentes proyectos y concursos internacionales. Un ejemplo de ello es el II Premio obtenido en el año 2008 por la 
organización indígena de Huo'e por sus relatos qom. Estos textos fueron considerados el mejor material didáctico bilingüe según la Organización de Estados Iberoamericanos para la Educación, la Ciencia y la Cultura. Es decir, las organizaciones que durante décadas se conformaron para reivindicar la cultura indígena y el respeto por la educación, las tradiciones y los territorios, fueron parte de un proceso de reactualización identitaria.

\section{Las organizaciones indígenas: actores claves en los proyectos de desarrollo}

Fue a partir de la visibilización de las comunidades indígenas, obtenida por su trabajo en diferentes espacios y proyectos, que las organizaciones étnicas se consolidaron no solo territorialmente sino también a nivel internacional, transformándose en actores claves de los organismos internacionales. En este contexto llegan a Pampa del Indio las agencias de crédito y las organizaciones no gubernamentales solventadas con ingresos europeos. Un primer proyecto aplicado en la localidad fue el de "Producción Ovina y Caprina" en el año 2007. El proyecto en cuestión era financiado por el Banco Mundial e implicaba la introducción y cría de ganado bovino y caprino en las localidades con población criolla y étnica.

El programa de "Desarrollo de Pequeños Productores Rurales en el Norte Argentino" se estableció a través del Ministerio de Agricultura, Ganadería y Pesca de la Nación a partir de la intervención del "Programa de Desarrollo Rural de las Provincias del Noreste Argentino" (PRODERNEA), siendo este último el mediador entre las organizaciones étnicas y criollas y los organismos nacionales e internacionales. Además, PRODERNEA trabajó coordinadamente con organizaciones no gubernamentales del orden local como ENDEPA e INCUPO, dado que estas eran las que tenían mayor presencia y posibilidad de generar un seguimiento más exhaustivo en la aplicación del programa por su estrecha vinculación con las comunidades en el territorio. Dicho programa tenía como fin generar alternativas productivas en las comunidades para fortalecer la cría de ganados, la intensificación de los cultivos, y la cría de abejas, entre otras. Para su aplicación se obtuvo la autorización del 'Enauac, de la organización de Huo'o, la asociación Cacique Indígena y la Comisión de Restitución Territorial, que trabajaban en la zona de Pampa Chica y Campo Cacique.

Este proyecto presentó algunas inconvenientes en su concreción. Algunas de estas dificultades se debían a no conocer la distinción entre criollos e indígenas; según la auto adscripción de sus integrantes se entregaba ganado bovino a los criollos, y ganado caprino a los aborígenes. Esta diferenciación no solo generaba malestar entre las organizaciones étnicas sino también la imposibilidad de ampliar su producción. La excusa que tenían los técnicos para explicar esta división del ganado era que las comunidades indígenas no estaban habituadas a trabajar en su economía tradicional con estos animales y podría generarse un desequilibrio cultural. La realidad es que desde el siglo XVII las comunidades de la región trabajaron con los animales que introdujeron los españoles, sobre todo el ganado bovino y caprino. Es decir, la exotización de los indígenas estaba en la base de las formas de pensar y ejecutar estas políticas (Castelnuovo, 2015; Iñigo Carrera, 2012a, 2013) ${ }^{4}$. Otro problema fue que PRODERNEA dejó de trabajar en el proyecto a fines del primer 
año de aplicación, con lo cual tuvo que reorganizarse el seguimiento a partir de los técnicos locales de las otras ONGs. Como consecuencia de esta mala ejecución se entregaron a la población solo cincuenta cabezas de ganado, cuando en la planificación se contemplaba la entrega de doscientas. Además, el proyecto no analizaba las condiciones de crisis económica estructural que había en la localidad. Por otra parte, la falta de pasturas y de agua que posibilitaran un buen cuidado de estos animales hizo que prontamente se violara uno de los primeros principios que tenía el proyecto: no consumir a las crías. Asimismo, se comenzó a comercializar e intercambiar el ganado en ferias, más allá de la reglamentación que imponían los funcionarios y técnicos. A pesar de ello, las organizaciones indígenas lograron obtener mayor visibilización y la posibilidad de participar en futuros proyectos de desarrollo.

En este sentido, se aplicó en Pampa del Indio el proyecto "Infraestructura Vial para el Desarrollo del Norte Argentino" (de aquí en adelante proyecto de Desarrollo del NA), que a través de la intervención del Ministerio Nacional de Planificación Federal de Inversiones ${ }^{5}$, recibía fondos de organismos como el Banco Mundial y el Banco Interamericano de Desarrollo. El proyecto de Desarrollo del NA aplicado desde el año 2011 en la localidad, contemplaba obras hídricas y viales. El Estado Nacional a través del Ministerio de Planificación, decidió realizar mejoras edilicias en la Ruta Provincial № 3, que incluye el tramo Pampa del Indio-Villa Río Bermejito, con los fondos otorgados por el Banco Mundial. La inversión de 400 millones de dólares que se utilizaron para la realización de esta obra se ejecutó tras una evaluación social, en la cual miembros del Ministerio de Planificación relevaron la zona a intervenir y a las comunidades que se verían afectadas. El Banco Mundial, para entregar este tipo de préstamos establece una adenda donde especifica que cuando se realiza una obra que atraviesa una zona habitada por comunidades se debe trabajar con ellas. Catalina, coordinadora del proyecto de Desarrollo del NA, estableció en una entrevista que esta adenda que hace el Banco Mundial es en pos de proteger los intereses vinculados a las transferencias de capitales y arreglos entre los entes gubernamentales:

La asistencia de esta unidad deriva de los condicionamientos de los bancos para hacer los préstamos, no deriva de la intención de proteger el ambiente o los pueblos indígenas del área rural, más allá de lo que hace cualquier empresa de evitar que los rentistas 'molesten'. Esto era más riguroso porque el Banco Mundial tiene salvaguardas muy fuertes y está por encima de temas sociales e indígenas. (Catalina, Coordinadora del proyecto de Desarrollo del NA, marzo 2016)

En función de esta adenda que exige trabajar con las comunidades, el gobierno provincial contrató a la Fundación Noreste Argentino, que trabaja en la región desde la década de 1990. Dicha fundación de origen italiana y financiada por la Cooperación Italiana Nord Sud desde el año 1988 y por la Agencia de Naciones Unidas de la Comisión Europea, propuso trabajar con las comunidades étnicas través de un proyecto con las organizaciones de mujeres de las zonas afectadas por las obras. El Banco Mundial, a través del Ministerio de Planificación de la Nación y en coordinación con la Fundación NEA, comenzó a trabajar en la localidad de Pampa del Indio para, según su discurso, 'salvaguardar a las comunidades' y llevar a cabo, desde entonces, el proyecto denominado "Ruta de la Cultura Qom": 
La Iniciativa de la Ruta de la Cultura Qom (RCQ) es realizar un corredor de siete centros culturales manejados por mujeres artesanas qom - $10 \%$ de la población indígena del país pertenece a esta etnia -, repartidos a lo largo de la recientemente pavimentada ruta 3 en la provincia de Chaco, obra que se realizó como parte del Plan de Pueblos Indígenas del proyecto de Infraestructura Vial para el Desarrollo del Norte Argentino (20112016) financiado por el Banco Mundial. (Grupo Banco Mundial, 2016, p. 06)

La "Ruta de la Cultura Qom" tiene como objetivo la comercialización de la producción artesanal elaborada por las organizaciones étnicas de la zona. Dicho objetivo implicaba que, a partir de la producción de dichas artesanías, las comunidades indígenas se insertarían en el mercado, generando, de este modo, una independencia económica. Esto permitiría a las organizaciones qom entrar en un circuito de comercialización, no solo de sus productos sino también de su propia cultura a partir de proyectos de 'turismo étnico'.

Las siete localidades que atraviesa la "Ruta de la Cultura Qom" y en la que trabaja Fundación NEA con organizaciones indígenas son: Presidente Roca, Pampa Chica, Cuarta Legua, Pampa Grande, Fortín Lavalle, Villa Rio Bermejito, El Espinillo.

A partir del relevamiento, los técnicos de las organismos gubernamentales y no gubernamentales encontraron que en las localidades existían organizaciones étnicas ya conformadas y que había otros grupos indígenas que estaban comenzando a participar y construir una referencia colectiva en torno a diversas cuestiones sociales. En Pampa del Indio se vincularon con la organización Huo'o. Como desarrollamos anteriormente, dicha organización tiene sus antecedentes en el año 1985 a partir de la participación de organismos católicos. Huo'o se consolidó a lo largo del tiempo en vinculación con las otras organizaciones étnicas especialmente a partir de los contextos de protesta social. Esta organización, seleccionada por la Fundación NEA, no solo trabajaba en pos del reconocimiento cultural qom sino también en relación a la necesidad de hacer cumplir las leyes que establecían que la educación intercultural era un derecho tan importante como la restitución de tierras. En este contexto la organización indígena Huo'o se consolidó a nivel provincial (donde sus integrantes fueron reconocidas por su trabajo como "Ciudadanas llustres" por la Cámara de Diputados de la Provincia), y en el escenario nacional. Además, han sido premiadas internacionalmente por su trabajo en la recuperación cultural. En este sentido, podríamos decir que las políticas de desarrollo que buscaban instalarse en Pampa del Indio con el objetivo de 'consolidar a las organizaciones indígenas' ya tenían un importante nivel de concreción dados los antecedentes que tenía la organización Huo'o en la localidad. Cabe aclarar que entre una de las finalidades del proyecto que presentó la Fundación figura la construcción de un salón comunitario que ya existía, dado que había sido creado por esta organización étnica a fines de la década de 1980 junto a las monjas de ENDEPA e INCUPO.

Según el informe que realizaron los técnicos del Banco Mundial los objetivos a alcanzar con el proyecto de la Ruta de la Cultura Qom en las comunidades fueron: construcción de centros comunitarios; realización de talleres sobre técnicas artesanales, inserción en los procesos productivos, elaboración de charlas sobre el derecho de la mujer y salud refractiva, y formación sobre asociaciones de 
mujeres, entre otros. Más allá de estos objetivos, la organización étnica ya venía trabajando en la comercialización de artesanías, dado que esto históricamente fue parte del sustento económico de sus familias y es lo que permitió el trabajo cotidiano de la organización, con lo cual este punto también estaba resuelto antes de la intervención de la Fundación. Respecto a la realización de charlas educativas sobre salud, la organización indígena en coordinación con la Universidad del Nordeste venía realizando talleres de capacitación acerca de la ley № 26.485 de protección integral de la mujer. En dichas charlas miembros de Huo'o no solo se reunían con los habitantes de la comunidad sino también proponían traducir las leyes en lengua toba.

En una entrevista que le realizamos a Flavia, presidenta de la Fundación NEA, se nos explicó el trabajo que desarrollaron desde la Fundación a partir del proyecto de la Ruta de la Cultura Qom y cómo fue la participación que tuvo dicha ONG en el trabajo que vienen realizando en el territorio:

Desde la fundación venimos trabajando en proyectos de desarrollo con el foco en las mujeres hace un tiempo, con el empoderamiento de las mujeres indígenas en particular, y a través de distintas estrategias para generar ingresos para lograr el empoderamiento para dinamizar esos procesos. Nosotros tenemos como objetivo el empoderamiento de las mujeres, o sea nosotras consideramos que para trabajar todos los otros temas tiene que haber un sujeto fortalecido que pueda exigir políticas públicas. Después ellas tienen que ir a la mesa de salud a los problemas donde se plantean esas cosas. Se pueden hacer talleres de salud o violencia, pero no se va a generar ningún cambio. El empoderamiento es nuestro foco principal de acción, ahora no nos dispersamos. (Flavia, presidenta de la Fundación NEA, marzo 2016)
Como vimos anteriormente, la organización étnica Huo'o trabaja desde finales de la década de 1980 con el objetivo de lograr su reconocimiento cultural, la preservación de sus tradiciones e historia. Esta organización también se vinculó con otros movimientos indígenas y criollos de la zona para tener en la localidad una educación bilingüe e intercultural, tal como establece la Constitución Nacional. Pero las demandas que lleva a cabo no solo se enmarcan en cuestiones culturales sino también territoriales. Los recursos naturales, así como la tierra, son un bien que disputan las organizaciones indígenas históricamente con los empresarios que se las arrebataron. En este sentido una de las actividades que realizaron dichas organizaciones étnicas fue la confección de un mapa donde plasmaron las zonas de marisca y de pesca, sus lugares sagrados y cementerios, entre otros. Tal trabajo toponímico lo realizaron integrantes indígenas, sobre todo los miembros de Huo'o, junto a la colaboración de un antropólogo chileno; Burgos, de la Escuela de Antropología de la Universidad Bolivariana y a quien pudimos entrevistar, nos explicó cómo fue el proceso de confección del mapa:

Cuando llegué a Pampa del Indio me pidieron hacer una herramienta de reivindicación territorial para INCUPO. No era una inquietud de las organizaciones. Era una herramienta para profesionales y yo creía que había que hacerla con las comunidades, y esa fue mi primera pelea. Hicimos un taller de mapeo intercultural para generar la reivindicación territorial. Se empezó con construir como ellos ven el territorio, como comprenden la geografía, y luego pasar a un ámbito educativo, para pasar a las generaciones futuras una herramienta que pueda ser leída y abordada por niños, como por jóvenes, como por mujeres y hombres. (Entrevista a Carlos Burgos, octubre 2016) 
Este mapa, que para la organización significó un arduo trabajo con todas las comunidades indígenas de la zona, y que expresa cómo fue el territorio antes de las ocupaciones de los empresarios agro-ganaderos, fue utilizado en el marco del proyecto de "La Ruta de la Cultura Qom". Los mapas en la banquina a lo largo de la Ruta Provincial № 3 señalan en el camino dónde habitan las comunidades indígenas, cómo se llaman dichas zonas en lengua toba y dónde se encuentran los lugares sagrados y cementerios étnicos, tal cual figura en el mapa que ellos confeccionaron junto a Burgos. Ahora bien, ni la Fundación NEA ni el Ministerio de Planificación emplazaron estos mapas en la localidad con el fin de reconocer, reivindicar y restituir el territorio que pertenece históricamente a las comunidades, sino con el objetivo de estimular el turismo rural y étnico y convertir a los indígenas en un atractivo turístico en sí mismo.

Sin embargo, para las comunidades observar sus propios mapas, realizados colectivamente en espacios de debate y reflexión sobre la memoria histórica y social, significa una reivindicación histórica y la posibilidad de disputar dichos espacios. Es decir, este proyecto de vialidad financiado por un organismo de renombre internacional como es el Banco Mundial generó la implantación de un proyecto turístico y la exotización de los indígenas, mostrando la 'otredad étnica' sin tener en cuenta las reivindicaciones, los derechos y las luchas que llevan a cabo las organizaciones.

Esta adenda del Banco Mundial de trabajar con las comunidades indígenas que se ven afectadas por la obra vial posibilitó que, a partir de la intervención de la Fundación, los integrantes de las organizaciones -en este caso las de Huo'o- hagan visible su lucha a nivel internacional. En este sentido, miembros de la organización viajaron a Italia y a la India en el marco de un programa llamado "Slow Food". Dicho encuentro internacional, donde participaban integrantes de comunidades 'exóticas' de diferentes partes del mundo, tenía como objetivo generar actividades en pos de desarrollar estrategias nutricionales contra la comida chatarra o 'comida rápida'. En este contexto, la participación de Huo'o contribuyó enseñando la cantidad de alimentos naturales y nutritivos que se pueden hacer con una semilla nativa como la algarroba.

Las integrantes de la organización étnica utilizaron este espacio colmado de representantes de diferentes organismos, para resignificar la actividad relatando sus experiencias no solo como 'cocineras autóctonas' sino también denunciando lo que ocurre en la región chaqueña a partir de la expansión sojera especialmente centrándose en el uso de agroquímicos. Las participantes enfatizaron que estos productos tóxicos no solo generan enfermedades en las personas y animales sino también extinguen la vegetación autóctona.

Cabe aclarar que dichos agros tóxicos son utilizados sobre todo en las plantaciones de soja de la región por diferentes productores. Un empresario apodado "el rey de la soja", es uno de los dueños de mayores extensiones de tierras en el negocio del agro, y utiliza dichos productos en sus cultivos. Varsovia forma parte de una asociación civil llamada Asociación Civil para la Integración y el Desarrollo (ACID) que trabaja en coordinación con la Fundación NEA en el proyecto de la Ruta de la Cultura Qom. El "rey de la soja" posee más de 300.000 mil hectáreas cultivadas en Argentina y otras tantas en el exterior y fue el comprador de la 
planta Agrofina que sintetiza y formula fitosanitarios de alto valor. Los fitosanitarios son productos utilizados para combatir los parásitos y enfermedades que puedan tener los cultivos como también exterminar las hierbas y plantas que los rodeen. Al utilizar este agente en el medio ambiente, se generan cambios en la atmósfera, el suelo y el agua. Está comprobado que su uso contamina y produce trastornos en la salud de los seres vivos. En un diario de Valencia, España, relatan cómo un grupo de expertos denunciaron, a partir de estudios del suelo y el agua, sus riesgos:

La comisión de las Cortes que investiga la contaminación de los acuíferos, un problema que afecta especialmente a las comarcas de la Safor y la Ribera continúa buscando explicaciones. En las sesiones celebradas esta misma semana, los expertos apuntaron al exceso de fertilizantes y productos fitosanitarios utilizados por los agricultores de la comarca como una de las principales fuentes de esta problemática. Los comparecientes hicieron hincapié en la falta de mecanismos de control durante décadas por parte de las administraciones públicas y reprocharon igualmente la escasa información sobre las consecuencias de esta práctica que llegaba al propio sector agrícola (...). «Si algo ha quedado claro es que el exceso de fertilizantes ha contribuido al aumento de nitratos disueltos en el agua, los cuales generan diversas reacciones químicas en el organismo y pueden degenerar en problemas de salud», explicó uno de los especialistas. (Levante; el Mercantil Valenciano. 2016. "Un exceso en el uso de fitosanitarios contamina el agua en Safor y Ribera") ${ }^{6}$

Si bien la participación de Huo'o en dichos espacios, como el encuentro de Slow Food, sirvió para denunciar los conflictos que padecen en la localidad, también fue utilizada para obtener mayores beneficios económicos por parte del Banco Mundial y la Fundación NEA.

Nos interesa remarcar que dichos organismos de financiamiento internacional analizan esca- samente los procesos de organización indígena previos a su intervención en el territorio, generándose de este modo, una mirada parcial acerca de las trayectorias de estas comunidades. Este aparente desconocimiento de la historia de los pueblos, dada su complejidad y amplia red de actores, puede conducir a una parcial mirada a la hora de planificar soluciones de las problemáticas locales (como son la falta de acceso al agua o los conflictos territoriales). Catalina, integrante del proyecto de Desarrollo del NA, describió al respecto que la situación local a partir del cambio de gobierno en diciembre del año 2015 significó la paralización de las obras planificadas:

Iban a hacer un acueducto, era un proyecto que iba a dotar a mucha gente y estaba empezando la ejecución que se estaba haciendo con Fundación NEA, pero que está parado y no alcanzaron a hacer el acueducto que va de Roca a Pampa del Indio haciendo activaciones comunitarias por las comunidades y después un montón de pueblos hacia el sur. (Catalina, proyecto de Desarrollo del NA, marzo 2016)

Un tiempo después Cintia, técnica de la Fundación NEA encargada de la zona de Pampa del Indio, explicaba en una entrevista cuál era la situación que atravesaba el planeamiento de esta obra:

Se presentó una ampliación del acueducto porque no se aprobó porque se extendía del presupuesto y el gobierno provincial ahora tiene que ver de dónde se saca el financiamiento... el acueducto va de Castelli a Miraflores y ahora falta de este lado, pero no se aprobó, ahora hay que ver si el gobernador encuentra otra línea de financiamiento. (Cintia Fundación NEA, abril 2016)

En las entrevistas realizadas a los miembros de la organización étnica -al preguntárseles sobre los cambios en sus cotidianidades o 
acerca de los beneficios obtenidos a partir de la participación en las políticas de desarrollo-, las respuestas que daban referían a la posibilidad de viajar y conocer otras organizaciones con historias similares y con las que compartían las mismas reivindicaciones. Asimismo, para dichos integrantes la intervención de los organismos significaba la posibilidad de viajar al exterior para dar a conocer la situación y visibilizar sus problemáticas. Sin embargo, a pesar de estos beneficios, los entrevistados nunca vincularon su participación en estos programas con un mayor empoderamiento -que era uno de los objetivos de la Fundación NEA en el proyecto de la Ruta de la Cultura Qom- ni con mejoras en relación a la infraestructura (vial o hídrica) que beneficiaran a la comunidad.

Como analizamos, los proyectos de financiamiento internacional que se ejecutan en los territorios con altos grados de conflicto territorial y con dificultades de acceso a los recursos como el agua, no tienen en cuenta dichas problemáticas y, por ende, tienen éxitos parcializados (Escobar, 1998). Otro problema asociado a los organismos como la Fundación NEA es la apropiación que realizan del trabajo generado por las organizaciones étnicas, sin reconocer su historia local. Un ejemplo de ello es lo ocurrido con el caso del salón comunitario de Pampa Grande a partir de la intervención de la empresa Samsung. Dicha empresa, tras donar algunas tablets y una computadora de escritorio, determinó que el centro comunitario se llamaría "Samsung namum village". Esta iniciativa elimina la mirada y cosmovisión de la organización Huo'o, que trabaja en ese salón con el objetivo de preservar su cultura frente al avasallamiento occidental. Cintia, de la Fundación NEA, se explaya sobre este tema en una entrevista:
Entre los proyectos está Samsung porque aparte lo que pretende con el Nanum es que sea como un ojo de la comunidad. En ningún lado dice que es como un ojo, pero eso se espera. Nanum es un proyecto con Samsung desde la Fundación que tiene como objetivo generar una red de productores, 'El futuro está en el monte' se llama en la Fundación. Negocios en red, lograr desarrollo desde el monte nativo y desde la revalorización de los saberes tradicionales que tienen los productores (...) el objetivo de estos centros es acercar innovación tecnológica a las comunidades aisladas del Gran Chaco. (Cintia, Fundación NEA, marzo 2016)

En este contexto observamos cómo las políticas de desarrollo fueron implementadas en el territorio con el objetivo de incrementar la producción local y si bien, retoman aqueIlas historias y trayectorias locales para efectuar espacios de debate, talleres o consultan, terminan generando instancias donde estos hechos son retomados escasamente o no son considerados. En este sentido, tales proyectos parecerían tener resultados con instancias de desigualdad, exclusión y exotización de las comunidades étnicas con los que se vinculan, pero tambien, encontramos instancias de organización y politización de estos actores a partir de procesos de reactualización y revalorización.

\section{Consideraciones finales}

Como analizamos a lo largo de este artículo, el contexto en la República Argentina se modificó a través del tiempo. Desde la década de 2000 en adelante el escenario internacional y local provocó cambios a nivel económico y por lo tanto a nivel social. En este sentido, el estallido social se visualizó sobre todo durante el año 2001. Concepciones como "defender lo nacional frente a la extranjerización", "reivindicar la cultura y las tradiciones", 
y "respetar los derechos y revalorizar lo local", comenzaron a tomar mayor visibilidad en esta década. La participación popular en espacios de política, los debates en torno a cuál era la mejor forma de vivir en el país, de pagar las deudas o la necesidad de reclamar por un trabajo, eran las actividades que llevaban a cabo las organizaciones que durante los años de crisis se nutrieron de herramientas para ser reconocidas por los gobiernos provinciales, nacionales e internacionales.

Vimos a lo largo del artículo como las organizaciones indígenas y de pequeños productores que se conformaron en Pampa del Indio, estuvieron muchas veces acompañadas por las iniciativas de los organismos religiosos. Con el tiempo, los integrantes de las organizaciones indígenas comenzaron a vincularse con otros actores a partir de la participación en programas sociales o como contraprestación para cobrar un subsidio. Otra cuestión que analizamos es que dichas organizaciones comenzaron a tener sentidos de pertenencia que construyeron desde las vivencias personales y locales: las problemáticas acerca del acceso a la educación intercultural de sus hijos, la falta de profesionales de la salud que trabajen con técnicas e insumos propios de la medicina indígena, o el acceso a sus tierras y sus recursos. En este sentido, las organizaciones agudizaron sus reclamos y comenzaron a intervenir políticamente en sus localidades para dar respuestas a estos problemas.
En este contexto, en Pampa del Indio, se han implementado diferentes políticas públicas a partir de la participación de fundaciones, organismos nacionales e internacionales, como es el caso de la Fundación Nea, INCUPO, ENDEPA, organismos pertenecientes al gobierno provincial y nacional, y el Banco Mundial. Hemos analizado a lo largo de este trabajo que iniciativas vinculadas a los proyectos de desarrollo como La Ruta de la Cultura Qom, Slow Food, Samsung Namún Village, generan contradicciones al articulares con los espacios de participación indígena cuyos intereses, muchas veces, se hallan contrapuestos. En este sentido, entendemos que el alcance final de estos proyectos busca generar un proceso de despolitización de los problemas sociales y económicos, así como una exotización aún mayor de las poblaciones con las que se trabaja. Sin embargo, como en todo proceso de participación, las organizaciones indígenas buscan obtener herramientas para llevar a cabo sus objetivos y adquirir de este modo, algún rédito que les sea favorable, aun en las negociaciones que desarrollan o tolerando la implementación de proyectos esencialistas y exotizantes. Nos parece importante mencionar que estas dinámicas, donde las organizaciones indígenas construyen herramientas para la reivindicación cultural a partir de la vinculación con fundaciones y organismos internacionales, son un aspecto para seguir profundizando en aportes futuros, teniendo en cuenta las articulaciones y tensiones que se presentan en estos escenarios de negociación que aquí buscamos describir. 


\section{Notas}

1 Tal como mencionamos al comienzo del artículo, los nombres de aquellos entrevistados, actores, fundaciones y organizaciones con los que trabajamos, fueron reemplazados por nombres ficticios para preservar su anonimato

2 Se denomina 'patria sojera' a la gran expansión de la frontera agraria de la soja entre los países de Brasil, Paraguay, Uruguay, Bolivia y Argentina, donde los cinco países contaban, aproximadamente, con 50 millones de hectáreas cultivadas con soja transgénica (Melón, 2014).

${ }^{3}$ La Orden de la Merced es una Orden religiosa católica que se fundó en el año 1218 por San Pedro Nolasco con el fin de lograr la redención de los cristianos cautivos en manos de los musulmanes. Los mercedarios se comprometen con un cuarto voto, añadido a los tradicionales de pobreza, obediencia y castidad de las demás órdenes de la religión católica, a liberar a otros más débiles en la fe, aunque su vida peligre por ello. Los mercedarios consideran que deben liberar a las comunidades indígenas dado que a estas se les negó

\section{Referencias bibliográficas}

Acuña, C. (Ed.). (2014). El Estado en acción: fortalezas y debilidades de las políticas sociales en la Argentina. Buenos Aires: Siglo Veintiuno Editores Argentina.

Aranda, D. (2012). La Patria Sojera. En: Revista MUU. Recuperado de: http://www.lavaca.org/notas/patria-grande-y-sojera/ (fecha de consulta: 28 de septiembre de 2016)

Bartolomé, M. (2003). Los pobladores del "desierto". En Amérique Latine Histoire et Mémoire. Les Cahiers ALHIM. En línea: http:// journals.openedition.org/alhim/103 (26 de noviembre de 2017) (2004). Movilizaciones étnicas y crítica civilizatoria. Un cuestionamiento a los proyectos estatales en América Latina. Perfiles Latinoamericanos, (24), 85-105

(2008). Oguerojerai (desplegarse). La etnogénesis del

Pueblo Mbya-Guaraní. Ilha Revista de Antropología, 10(1), 105-140. (2010). Interculturalidad y territorialidades confrontadas en América Latina. Runa, 31(1), 09-29.

Bello, B. M. (2004). Etnicidad y ciudadanía en América Latina: la acción colectiva de los pueblos indígena. Santiago de Chile: Cepal.

Bello, M. \& Rangel, M. (2002). La equidad y la exclusión de los pueblos indígenas y afrodescendientes en América Latina y el Caribe. Revista de la CEPAL.

Bengoa, J. (2000). La emergencia indígena en América Latina. Fondo de Cultura Económica. Santiago.

(2009). ¿Una segunda etapa de la Emergencia Indígena en América Latina? Cuadernos de antropología Social, (29), 07-22.

Blanco, M. R. (2005). Propuesta para un análisis del movimiento indígena como movimiento social. Política y Sociedad, 42(49-62)

Braticevic, S. (2011). El papel de las ONG en proyectos de su cultura, y la pobreza espiritual de ellos radica en dicha negación.

${ }^{4}$ Encontramos una gran cantidad de literatura que aborda esta problemática durante los últimos años, pero por motivos de espacio no profundizaremos en este artículo en dicha temática.

${ }^{5}$ El Ministerio de Planificación Federal e Inversiones fue uno de los ministerios de Argentina durante diversas gestiones presidenciales. Hasta la asunción de Mauricio Macri como presidente de la Nación (2015), el Ministerio estaba a cargo de la conducción de Julio De Vido, ministro que se mantuvo en su cargo durante los gobiernos de Néstor Kirchner (2003-2007) y Cristina Fernández de Kirchner (2007-2011 y 2011-2015).

${ }^{6}$ Levante; el Mercantil Valenciano. "Un exceso en el uso de fitosanitarios contamina el agua en Safor y Ribera". Fecha de publicación: 04 de diciembre de 2016. Página Online: http://www. levante-emv.com/safor/2016/12/04/exceso-fitosanitarios-contaminaagua-safor/1500327.html

desarrollo en una formación social de fronteras. El caso del programa DIRLI en el oeste formoseño. En: Intersecciones en antropología, 1(12), 135-146

Campos, L. (2006). Descubriendo la multiculturalidad. El caso chileno. Presentación: Multiculturalismo e interculturalidad en América Latina Alicia M. Barabas, 33.

Carrasco, M. \& Briones, C. (1996). La tierra que nos quitaron. IGWIA, Buenos Aires.

Carrera, V. I. (2012). Movilización indígena en el noreste formoseño. Notas a propósito de un caso. Relaciones-Sociedad Argentina de Antropología, 37(2), 457-462.

Castelnuovo, N. (2016). Etnografiando negociaciones, acuerdos y alianzas entre políticos provinciales y ONG en el noroeste argentino. Espiral (Guadalajara), 23(65), 165-200.

(2014). Mujeres guaraníes y desarrollo en el noroeste argentino. Buenos Aires: Antropofagia.

(2015). El fomento de artesanías indígenas chané: ¿fortalecimiento de identidades o reproducción de desigualdades? "La diversidad como recurso. Producción artesanal chané destinada a la comercialización e identidad". Benedetti, Cecilia. Antropofagia. RUNA, archivo para las ciencias del hombre, 36(1), 127-128.

Castilla, M. (2017). De lo global a lo local: políticas públicas, legislaciones y surgimiento de organizaciones étnicas en Pampa del Indio, Chaco. Revista Papeles de Trabajo. (34), 29-44.

Constitución Nacional de la República Argentina. (1994). Argentina.

De La Cruz, L. (1997). Y no cumplieron. Proyecto de Desarrollo Agroforestal en Comunidades Rurales del Noroeste Argentino y 
Fundación para el Desarrollo Agroforestal de las Comunidades del Noroeste Argentino, Formosa.

Escobar, A. (1998). La invención del tercer mundo: construcción y deconstrucción del desarrollo. Bogotá: Norma.

Espinoza, C. (2017). Procesos etnopolíticos en la transición democrática chilena. Gobiernos locales y la vía política mapuche. Cuadernos de antropología social, (45), 21-36

García, A. \& Valverde, S. (2007). Políticas estatales y procesos de etnogénesis en el caso de poblaciones mapuche de Villa La Angostura, provincia de Neuquén, Argentina. Cuadernos de antropología social, (25), 111-132.

Grupo del Banco Mundial. (2016). Caminos hacia el aumento de la capacidad de acción y decisión de las mujeres. Iniciativa de la ruta de la cultura qom, provincia de chaco - argentina. Proyecto de infraestructura vial para el desarrollo de norte grande (2011-2016). Recuperado de: www.worldbank.org: (Fecha de consulta: 14 de agosto 2016).

Hecht, A. (2014). Escolarización de hablantes de Toba/Qom: Cruces entre políticas, estadísticas y trayectorias escolares. Papeles de trabajo - Centro de Estudios Interdisciplinarios en Etnolingüística y Antropología Sociocultural, (27), 103-127. Recuperado en 29 de diciembre de 2017, de http://www.scielo.org.ar/scielo.php?script=sci_ arttext\&pid=S1852-45082014000100005\&lng=es\&tlng=es.

(2006). De la familia Wichí a la escuela intercultural bilingüe: procesos de apropiación, resistencia y negociación (Formosa, Argentina). Cuadernos Interculturales, 4(6), 93-113

Instituto Nacional de Estadísticas y Censos (INDEC) (2001). Censo Nacional de Población, Hogares y Viviendas, Republica Argentina.

(2010). Censo Nacional de Población, Hogares y Viviendas, Republica Argentina.

Iñigo Carrera, V. (2012a). La producción de la "cultura aborigen" en el Chaco argentino: de naturalezas, estigmas, exotismos y fetichismos. Interseções: Revista de Estudos Interdisciplinares, 13(1), 7-25 (2012b). Movilización indígena en el Chaco argentino. Acción y conciencia políticas entre los qom del este de Formosa. Indiana, (29).
(2013). Movilizaciones indígenas e identidades en disputa en la Argentina. Revista Colombiana de Antropología, 49(1), 301-307.

Leoni de Rosciani, M. (1992). Los comienzos del Chaco provincializado (1951-1955). Rcia. IIGHI-CONICET. Cuadernos de Geohistoria, (26).

Melón, D. (coord) (2014). La patria sojera. El modelo agro sojero en el Cono Sur. Buenos Aires: El Colectivo.

Oliveira, J. P. (2010). ¿Una etnología de los indios misturados? Identidades étnicas y territorialización en el Nordeste de Brasil. Desacatos, (33), 13-32.

Provincia del Chaco. (1987). Ley del Aborigen Chaqueño $N^{\circ}$ 3258, Chaco. Argentina.

Radovich, J. C. \& Balazote, A. (2009). El pueblo mapuche contra la discriminación y el etnocidio. Ghioldi, Gerardo (Comp.). Historia de las familias mapuche Lof Paichil Antriao y Lof Quintriqueo de la margen Norte del lago Nahuel Huapi. Archivos del Sur-Villa La Angostura.

Stavenhagen, R. (2002). Identidad indígena y multiculturalidad en América Latina. Araucaria, 4(7).

(2005). La emergencia de los pueblos indígenas como nuevos actores políticos y sociales en América Latina. En Escárzaga, F. \& Gutiérrez, R. (Coord.) Movimiento indígena en América Latina: resistencia y proyecto alternativo (pp. 49-61). México: Universidades Autónomas de Puebla y México,

Trinchero, H. (2009). Pueblos originarios y políticas de reconocimiento en Argentina. Papeles de trabajo-Centro de Estudios interdisciplinarios en Etnolingüística y Antropología Sociocultural, (18), 0-0.

Trinchero, H., Muñoz, L. \& Valverde, S. (2014). Pueblos indígenas, Estados nacionales y fronteras. CLACSO.

Valverde, S. (2013). De la invisibilización a la construcción como sujetos sociales: el pueblo indígena Mapuche y sus movimientos en Patagonia, Argentina. Anuario Antropológico, (I), 139-166.

Weiss, L., Engelman, J. \& Valverde, S. (2013). Pueblos Indígenas Urbanos en Argentina: un estado de la cuestión. Revista Pilquen, 16(1), 00-00. 